mediated by complement bypass systems. Fourth, and probably most important, the type of assay represents the wave of the future in detecting the activity of the complement system in human disease. As these more informative detection systems (like the one used in this report) come into routine clinical use, other examples of these bypass type pathways will likely be uncovered. For human diseases, these more specific and quantitative assay systems will establish which pathway of complement activation is playing a role in disease and elucidate which one to modulate with therapeutic agents.

Finally, a word of caution is in order. These bypass pathways are often not considered by investigators attempting to define the role of the complement system in disease states. For example, C4-deficient animals are widely used to rule out a contribution of the classical pathway and/or lectin pathway in mouse models of human disease. One must be wary of such interpretations in view of bypass cascades that become operative in "deficient" states. Thus the natural maturation of an antibody response to an infectious organism (i.e., to go rapidly into antibody excess) is all that is necessary to trigger these more ancient bypass pathways. Using our current methods, such pathways are not analyzed in clinical medicine or in animal models of human disease.

Address correspondence to: John P. Atkinson, Washington University School of Medicine, 660 South Euclid Avenue, Box 8045, St. Louis, Missouri 63110, USA. Phone: (314) 362-8391; Fax: (314) 362-1366; E-mail: jatkinso@im.wustl.edu.

1. May, J.E., and Frank, M.M. 1973. A new complement-mediated cytolytic mechanism-the C1-bypass activation pathway. Proc. Natl. Acad. Sci. U. S. A. 70:649-652.

2. May, J.E., and Frank, M.M. 1973. Hemolysis of sheep erythrocytes in guinea pig serum deficient in the fourth component of complement. I. Antibody and serum requirements. J. Immunol. 111:1661-1667.

3. May, J.E., and Frank, M.M. 1973. Hemolysis of sheep erythrocytes in guinea pig serum deficient in the fourth component of complement. II. Evidence for involvement of $\mathrm{C} 1$ and components of the alternate complement pathway. J. Immunol. 111:1668-1676.

4. Matsushita, M., and Okada, H. 1986. Alternative complement pathway activation by $\mathrm{C} 4 \mathrm{~b}$ deposited during classical pathway activation. J. Immunol. 136:2994-2998.

5. Farries, T.C., Knutzen Steuer, K.L., and Atkinson, J.P. 1990. The mechanism of activation of the alternative pathway of complement by cell-bound $\mathrm{C} 4 \mathrm{~b}$. Mol. Immunol. 27:1155-1161.
6. Knutzen-Steuer, K.L., et al. 1989. Lysis of sensitized sheep erythrocytes in human sera deficient in the second component of complement. J. Immunol. 143:2256-2261.

7. Selander, B., et al. 2006. Mannan-binding lectin activates $\mathrm{C} 3$ and the alternative complement pathway without involvement of C2. J. Clin. Invest. 116:1425-1434. doi:10.1172/JCI25982.

8. Seelen, M.A., et al. 2005. Functional analysis of the classical, alternative, and MBL pathways of the complement system: standardization and validation of a simple ELISA. J. Immunol. Methods. 296:187-198.

9. Deguchi, M., Gillin, F.D., and Gigli, I. 1987. Mechanism of killing of Giardia lamblia trophozoites by complement. J. Clin. Invest. 79:1296-1302.

10. Wagner, E., Platt, J.L., Howell, D.N., Marsh, H.C., Jr., and Frank, M.M. 1999. IgG and complementmediated tissue damage in the absence of C2: evidence of a functionally active C2-bypass pathway in a guinea pig model. J. Immunol. 163:3549-3558.

11. Traustadottir, K.H., Rafnar, B.O., Steinsson, K., Valdimarsson, H., and Erlendsson, K. 1998. Participation of factor $\mathrm{B}$ in residual immune complex red cell binding activity observed in serum from a C2-deficient systemic lupus erythematosus patient may delay the appearance of clinical symptoms. Arthritis Rheum. 41:427-434.

12. Klint, C., Gullstrand, B., Sturfelt, G., and Truedsson, L. 2000. Binding of immune complexes to erythrocyte CR1 (CD35): difference in requirement of classical pathway components and indication of alternative pathway-mediated binding in C2-deficiency. Scand. J. Immunol. 52:103-108.

13. Nolin, L., et al. 1979. Possible C1q bypass loop activation in the haemolytic uraemic syndrome. Clin. Exp. Immunol. 35:107-111.

\title{
The IL-23/IL-17 axis in inflammation
}

\author{
Yoichiro Iwakura and Harumichi Ishigame
}

Center for Experimental Medicine, Institute of Medical Science, University of Tokyo, Tokyo, Japan.

\begin{abstract}
IL-23 induces the differentiation of naive $\mathrm{CD} 4^{+} \mathrm{T}$ cells into highly pathogenic helper T cells (Th17/Th ${ }_{\text {IL-17) }}$ ) that produce IL-17, IL-17F, IL-6, and TNF- $\alpha$, but not IFN- $\gamma$ and IL-4. Two studies in this issue of the JCI demonstrate that blocking IL-23 or its downstream factors IL-17 and IL-6, but not the IL-12/ IFN $-\gamma$ pathways, can significantly suppress disease development in animal models of inflammatory bowel disease and MS (see the related articles beginning on pages 1310 and 1317). These studies suggest that the IL-23/IL-17 pathway may be a novel therapeutic target for the treatment of chronic inflammatory diseases.
\end{abstract}

\section{Th17/Th $\mathrm{IL}-17_{17}$ is a new CD4+ helper T cell subset that produces IL-17}

Upon antigenic stimulation, naive $\mathrm{CD}^{+}$ $\mathrm{T}$ cells differentiate into 2 subsets, Th1 and Th2 cells, characterized by different cytokine production profiles and effector

Nonstandard abbreviations used: CIA, collageninduced arthritis; IBD, inflammatory bowel disease; IL-1Ra, IL-1 receptor antagonist; R, receptor.

Conflict of interest: The authors have declared that no conflict of interest exists.

Citation for this article: J. Clin. Invest. 116:1218-1222 (2006). doi:10.1172/JCI28508. functions (Figure 1). Th1 cells produce large quantities of IFN- $\gamma$ and mediate cellular immunity while Th2 cells, which are involved in humoral immunity, primarily produce IL-4, IL-5, and IL-13. IL-12, a heterodimer of the p40 and p35 subunits, induces the differentiation of naive $\mathrm{CD}^{+}$ $\mathrm{T}$ cells into IFN- $\boldsymbol{\gamma}$-producing Th1 cells through activation of STAT4. IFN- $\gamma$ signals are transduced by STAT1, which activates a downstream transcription factor, T-bet, that enhances the expression of genes specific to Th1 cells. In contrast, IL-4 induces
STAT6 activation, promoting the expression of GATA-3, a transcriptional factor essential for both IL-4 production and Th2 cell differentiation. Recently, it was reported that $\mathrm{CD}^{+} \mathrm{T}$ cells isolated from the inflamed joints of patients with Lyme disease contain a subset of IL-17-producing $\mathrm{CD}^{+} \mathrm{T}$ cells that are distinct from those producing either IL-4 or IFN- $\gamma$ (Figure 1) (1). These IL-17-producing CD4 ${ }^{+} \mathrm{T}$ cells were dubbed Th17 or Th $\mathrm{T}_{\mathrm{IL}-17}$ cells $(2-4)$.

IL-17, a proinflammatory cytokine predominantly produced by activated $\mathrm{T}$ cells, enhances $\mathrm{T}$ cell priming and stimulates fibroblasts, endothelial cells, macrophages, and epithelial cells to produce multiple proinflammatory mediators, including IL-1, IL- 6 , TNF- $\alpha$, NOS-2, metalloproteases, and chemokines, resulting in the induction of inflammation $(5,6)$. IL-17 expression is increased in patients with a variety of allergic and autoimmune diseases, such as RA, MS, inflamma- 


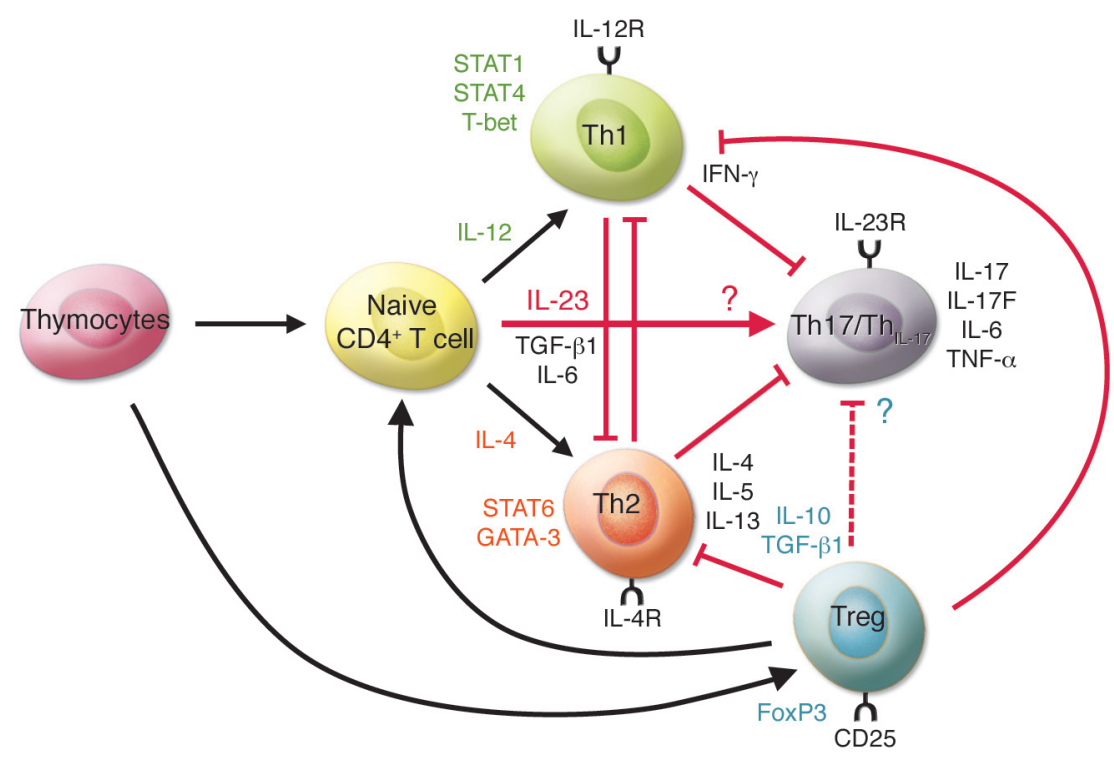

Figure 1

IL-23 promotes the development of an IL-17-producing CD4+ helper T cell subset. IL-23 induces the differentiation of naive CD4+ $\mathrm{T}$ cells into IL-17-producing helper T cells ( $\mathrm{Th} 17 / \mathrm{Th} / \mathrm{LL}-17)$ via mechanisms that are distinct from the Th1 and Th2 differentiation pathways. The transcriptional factors critical for the development of Th1 (STAT1, STAT4, and T-bet) and Th2 (STAT6) cells are not required for the induction of Th17/Th $\mathrm{IL}_{\mathrm{L}-17}$ cells. The transcriptional factor(s) essential for the development of Th17/Th $\mathrm{IL}_{\mathrm{L}-17}$ cells remain unknown. IFN- $\gamma$ and IL-4 antagonize each other in the differentiation of Th1 and Th2 cells and the promotion of their function. IFN- $\gamma$ also suppresses the differentiation of Th17/Th $\mathrm{IL}_{\mathrm{L}-17}$ cells by reducing IL-23R expression on CD4+ $\mathrm{T}$ cells. IL-4 also inhibits the development of Th17/Th $\mathrm{IL}_{\mathrm{L}-17}$ cells. It is not known, however, whether Th17/Th $\mathrm{IL}-17_{17}$ cells inhibit the development of Th1 and Th2 cells. Tregs, an immune-modulating subset of CD4+ $\mathrm{T}$ cells, suppress the differentiation and effector function of Th1 and Th2 cells. Recent studies suggest that Treg-derived TGF- $\beta$ induces the differentiation of Th17/Th $\mathrm{IL}_{17}$ cells from naive CD4+ T cells in the presence of IL-6 in vitro (26). However, the precise effect(s) of Tregs on $\mathrm{Th} 17 / \mathrm{Th}_{\mathrm{LL}-17}$ cells are as yet unknown.

tory bowel disease (IBD), and asthma, suggesting the contribution of IL-17 to the induction and/or development of such diseases. Supporting this, the involvement of this cytokine in such responses is demonstrated in animal models; autoimmune disorders such as collageninduced arthritis (CIA) and EAE, animal models for RA and MS, respectively, as well as allergic responses such as contact hypersensitivity, delayed-type hypersensitivity, and airway hypersensitivity were suppressed in IL-17-deficient (IL-17-/-) mice $(7,8)$ (Y. Komiyama et al., University of Tokyo, Tokyo, Japan, unpublished observations). Therefore, $\mathrm{Th} 17 / \mathrm{Th}_{\mathrm{IL}-17}$ cells are likely to play critical roles in the development of autoimmunity and allergic reactions.

The IL-23/IL-17, but not IL-12/IFN- $\gamma$, axis is critical for the development of autoimmune inflammatory diseases

The development of autoimmune diseases, such as RA, MS, and IBD, is thought to be mediated by Th1 cells because high levels of IL-12 and IFN- $\gamma$ are detected in inflammatory sites (9). In addition, treatment with mAbs against IL-12p40 suppresses such disease development in humans and animal disease models $(9,10)$. However, mice deficient in IL-12p35, IL-12 receptor $\beta 2$ (IL-12R $\beta 2$ ), IFN- $\gamma$, IFN- $\gamma \mathrm{R}$, or STAT1, which are critical molecules in IL-12/IFN- $\gamma$ mediated responses, exhibit an increased severity of diseases such as EAE and CIA (11-13). These observations are inconsistent with the notion that IL-12 is responsible for such disease development. As IL-23, an IL-12 family cytokine consisting of the p19 and $\mathrm{p} 40$ subunits, shares the p40 subunit with IL-12 and anti-p40 mAbs inhibit both cytokines, the involvement of IL-23 is suggested. Current evidence suggests that IL-23 is responsible for the differentiation and expansion of $\mathrm{Th} 17 / \mathrm{Th}_{\mathrm{IL}-17}$ cells from naive $\mathrm{CD} 4^{+} \mathrm{T}$ cells $(2,4,14)$.

In this issue of the JCI, Yen et al. report on their use of IL-23p19--- and IL-12p35-/- mice to demonstrate that IL-23, but not IL-12, is essential for the development of intestinal inflammation (15). They used IL-10-/- mice as a model of T cell-mediated IBD (16) and showed that the development of colitis was greatly suppressed by IL-23p19 deficiency but not IL-12p35 deficiency. Exogenous IL-23 administration accelerated the onset of colitis in $\mathrm{Rag}^{-/}$mice engrafted with IL-10-/-CD4+ $\mathrm{T}$ cells. Notably, IL-17 production was abolished in IL-23 $19^{-/-}$mice while IFN- $\gamma$ and IL-4 production were unaffected. IL-17 and IL- 6 expression by anti$\mathrm{CD} 3 \mathrm{mAb}-$ stimulated memory $\mathrm{CD} 4^{+} \mathrm{T}$ cells were augmented by IL-23, but not by IL-12, indicating that IL-23 can simulate memory $\mathrm{CD}^{+} \mathrm{T}$ cells. This result contrasts with the ability of IL-12 to stimulate naive $\mathrm{CD}^{+} \mathrm{T}$ cells. Moreover, treatment with both antiIL-6 and anti-IL-17 mAbs significantly ameliorated the severity of the intestinal inflammation induced by IL-23-treated $\mathrm{Rag}^{-/}$mice engrafted with $\mathrm{IL}-10^{-/-} \mathrm{CD} 4^{+} \mathrm{CD} 45 \mathrm{RB}^{\text {hi }} \mathrm{T}$ cells. These observations suggest that IL-17 and IL-6 derived from memory T cells are responsible for the development of intestinal inflammation downstream of IL-23.

Also in this issue of the JCI, Chen et al. report on their use of newly developed anti-IL-23p19 mAbs to demonstrate the involvement of IL-23 in EAE (17). The authors previously demonstrated that IL-23p $19^{-/-}$mice are resistant to EAE and CIA; production of IL-17, but not IFN- $\gamma$, is almost completely abolished in these mutant mice $(3,18,19)$. In contrast, IL-23p $35^{-/-}$mice exhibited decreased IFN- $\gamma$ production and increased IL-17 production, suggesting that IFN- $\gamma$ may suppress IL-17 production. Furthermore, IL-23induced but not IL-12-induced proteolipid protein peptide-specific $\mathrm{T}$ cells are highly encephalogenic (3). Consistent with these reports, the development of EAE was efficiently suppressed by treatment with anti-IL-23p19 or anti-IL-12/IL-23p40 $\mathrm{mAbs}$ by inhibiting infiltration of IL-17-, IFN- $\gamma$-, and TNF- $\alpha$-producing $\mathrm{CD} 4^{+} \mathrm{T}$ cells in the CNS. Disease severity correlated well with serum IL-17 levels; treatment with anti-IL-17 mAbs ameliorated the clinical disease score. Meanwhile, treatment with anti-IFN- $\gamma$ mAbs exacerbated disease, consistent with previous observations that IFN- $\gamma^{-/-}$and IFN- $\gamma \mathrm{R}^{-/-}$mice are highly susceptible to EAE $(12,13)$. Thus, it is clearly shown that the IL-23/IL-17 pathway, rather than the IL-12/IFN- $\gamma$ pathway, is critical for the development of autoimmune diseases. 

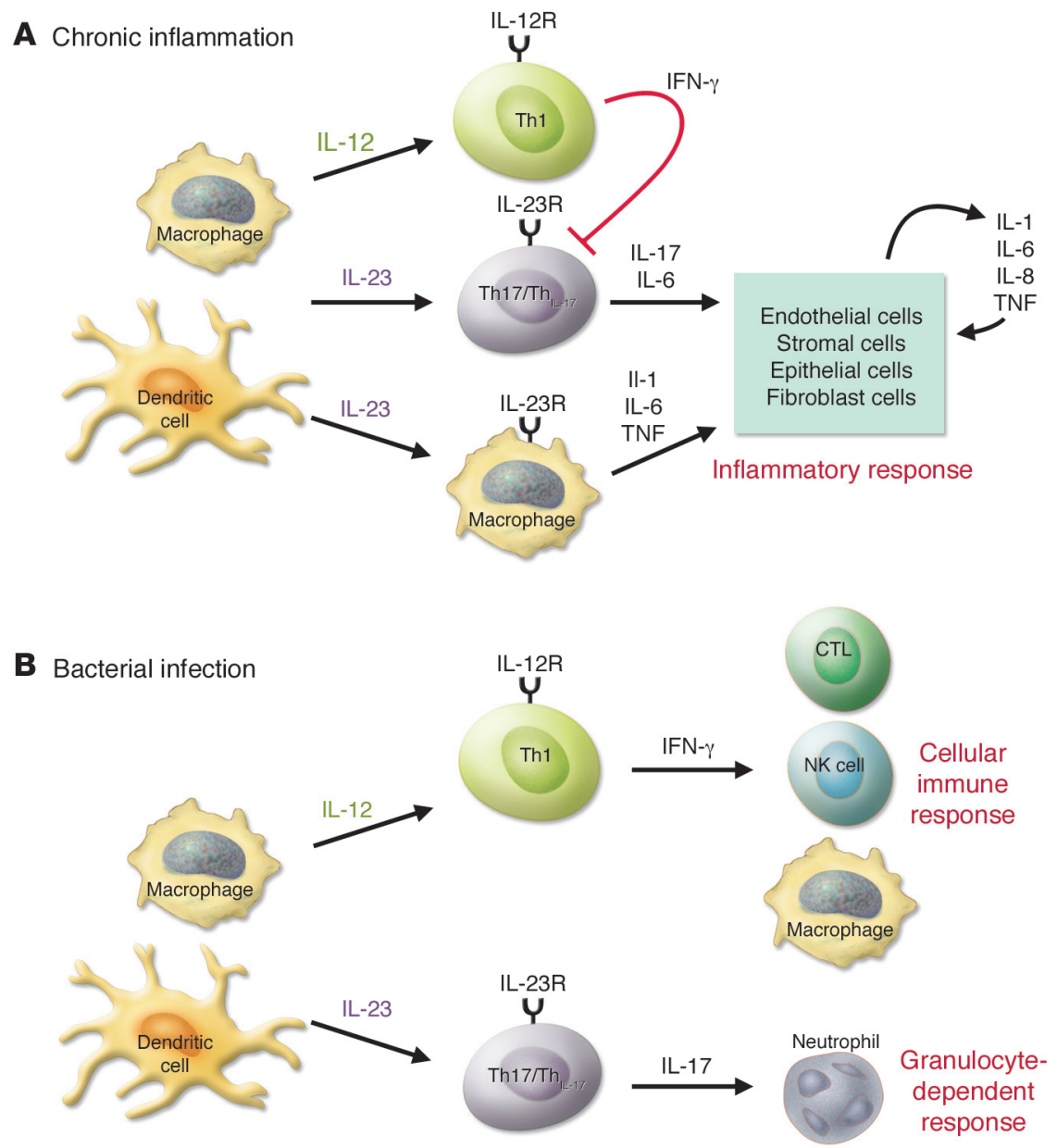

Figure 2

The role of the IL-23/IL-17 axis in inflammation and infection. The IL-23/IL-17 axis plays an important role in the development of chronic inflammation and in host defenses against bacterial infection. (A) In chronic inflammation, antigen-stimulated dendritic cells and macrophages produce IL-23, which promotes the development of Th17/Th $\mathrm{IL}_{\mathrm{L}-17}$ cells. Th17/Th $\mathrm{IL}-17$ cells produce IL-17, which enhances $T$ cell priming and triggers potent inflammatory responses by inducing the production of a variety of inflammatory mediators. IL-23 also acts on dendritic cells and macrophages in an autocrine/paracrine manner to stimulate the generation of proinflammatory cytokines, such as IL-1, IL-6, and TNF- $\alpha$. IL-12-stimulated Th1 cells produce IFN- $\gamma$ and suppress the differentiation of Th17/Th $\mathrm{IL}-17$ cells. Th1 cells may play an immunoregulatory, not a pathogenic, role in the development of chronic inflammation. (B) Upon bacterial infection, IL-23 is rapidly produced by activated macrophages and dendritic cells at the site of infection. IL-23 then activates local resident Th17/Th $\mathrm{IL}_{\mathrm{L}-17}$ cells and other IL-17-producing cells, such as CD8 ${ }^{+} \mathrm{T}$ cells and $\gamma \delta \mathrm{T}$ cells. Production of IL-17 by these cells induces G-CSF production from stromal cells. The IL-23/IL-17/G-CSF pathway augments neutrophil recruitment to the infection site, contributing to extracellular bacterial clearance. IL-23 also increases the production of IL-1, IL-6, and TNF- $\alpha$ in an autocrine/paracrine manner. In contrast, Th1 cells produce IFN- $\gamma$ and stimulate $\mathrm{CD} 8^{+}$cytotoxic T lympocytes, NK cells, and macrophages. IFN- $\gamma$ enhances antigen presentation by inducing expression of MHC molecules and activates cells to produce cytolytic molecules, including perforin and granzyme, which promote the elimination of intracellular bacteria.

\section{IL-23 causes inflammation through IL-17-dependent and IL-17- independent pathways}

Anti-IL-17 treatment did not prevent the onset and relapse of EAE with the same efficiency as anti-IL-23p19 or anti-
IL-12/IL-23p40 treatment, suggesting that the effects of IL-23 cannot be explained by the action of IL-17 alone. We also observed that EAE progression is only partially suppressed even in IL-17/- mice, suggesting the involvement of additional factors
(Komiyama et al., unpublished observations). The transcription factor T-bet is responsible for enhancing IFN- $\gamma$ production and increasing IL-12R $\beta 2$ expression (20). In contrast to IFN- $\gamma^{-/-}$mice, T-bet ${ }^{-/}$ mice are highly resistant to EAE development (11). As Th17/Th $\mathrm{IL}_{17}$ cells were present in T-bet ${ }^{-/-}$mice, Chen et al. suggested that $\mathrm{Th} 17 / \mathrm{Th}_{\mathrm{IL}-17}$ cells are not sufficient to induce disease and that additional T-betdependent factors and/or cell populations play significant roles in disease pathogenesis (17). With respect to this, T-bet expression on dendritic cells, but not on T cells, is required for IL- 1 and chemokine production, contributing to the development of collagen antibody-induced arthritis (21). In the current studies, Chen et al. (17) suggested that IL-23 may directly activate a subset of macrophages and dendritic cells expressing IL-23R, resulting in the production of inflammatory mediators, such as TNF- $\alpha$ and IL-1 $(18,22)$. However, as progression of diseases such as EAE and CIA is only minimally affected in TNF- $\alpha^{-/-}$mice and anti-TNF- $\alpha$ treatment of wild-type mice results in more severe MS (23), TNF- $\alpha$ is not critical for the development of this disease. In contrast, IL-6/- and IL-1/mice are significantly resistant, and IL-1 receptor antagonist-deficient (IL-1 $\mathrm{Ra}^{-/-}$) mice are more susceptible to $\operatorname{EAE}(24,25)$. Thus, IL- 6 and IL-1 likely play important roles in the development of this disease. These observations suggest that IL-23 can induce chronic inflammation through 2 independent pathways: (a) activation of Th17/Th $\mathrm{IL}-17_{\text {cells; }}$ and (b) induction of IL-1 and IL-6 production via myeloid cell activation (Figure 2).

Additional IL-17 family molecules may also play an important role in the development of inflammatory diseases. The IL-17 family currently consists of 6 family members; some of these family members, such as IL-17F, share significant amino acid homology with IL-17 (also known as IL-17A), are induced by IL-23, bind the same receptor as IL-17, and are produced by Th17/Th $\mathrm{IL}-17$ cells. Thus, it is possible that these additional IL-17 members may be involved in inflammatory responses. It is important to elucidate the functions of these IL-17 family molecules in normal physiology and in disease pathogenesis.

\section{The Th17/Th $\mathrm{L}_{\mathrm{L}-17}$ differentiation mechanism is not yet known}

Naive $\mathrm{CD}^{+} \mathrm{T}$ cells isolated from mice deficient in STAT1, STAT4, or T-bet 
retained the ability to differentiate into Th17/Th $\mathrm{IL}_{17}$ cells in vitro following TCR stimulation in the presence of IL-23 (2). The generation of Th17/Th $\mathrm{TL}_{\mathrm{IL}} 17$ cells following immunization with antigen stimulation was also normal in mice deficient for STAT4, STAT6, or T-bet (4). Thus, these Th1- and Th2-specific transcriptional factors are not involved in the differentiation of Th17/Th $\mathrm{IL}_{17}$ cells, indicating that the $\mathrm{Th} 17 / \mathrm{Th}_{\mathrm{IL}-17}$ lineage is independent from these classical Th cell lineages. In this issue, Chen et al. (17) demonstrate that T-bet ${ }^{-/}$lymph node cells produced IL-17 upon stimulation with anti-CD3 mAbs, consistent with previous reports $(2,4)$. Exogenous IL-23, however, did not further enhance IL-17 production in T-bet ${ }^{-/}$lymph node cells, suggesting that T-bet may influence IL-23 responsiveness during early $\mathrm{Th} 17 / \mathrm{Th}_{\mathrm{IL}-17}$ development (17). Thus, the transcriptional factors involved in $\mathrm{Th} 17 / \mathrm{Th}_{\mathrm{IL}-17}$ cell development still remain to be elucidated (Figure 1). With regard to this, STAT3 was recently implicated in the IL-23R signaling pathway (22).

As IL-12R $\beta 1$, the common subunit of IL-12R and IL-23R, is constitutively expressed in naive $\mathrm{CD}^{+}{ }^{+} \mathrm{T}$ cells, IL-12R $\beta 2$ and IL-23R expression are critical for the responsiveness to IL-12 and IL-23 and development of Th1 and Th17/Th $\mathrm{TL}_{17}$ cell lineages. Only memory and/or activated T cells express IL-23R; naive Th17/Th $\mathrm{TL}_{\mathrm{L}-17}$ progenitor cells are devoid of this molecule (22). However, when naive $\mathrm{CD}^{+} \mathrm{T}$ cells were stimulated with IL-23 in the presence of anti-IL- 4 and anti-IFN- $\gamma$ mAbs, a large IL-17-producing population was observed, indicating that IFN- $\gamma$ and IL-4 inhibit the differentiation of Th $17 / \mathrm{Th}_{\mathrm{IL}-17}$ cells from naive $\mathrm{CD}^{+} \mathrm{T}$ cells. IFN- $\gamma$ and STAT1 signaling inhibit the differentiation by downregulating the expression of IL-23R (2). Although IL-4 also inhibits Th17 cell expansion, the mechanism governing this suppression is not known $(2,4)$. Thus, the identification of the signals that induce IL-23R expression on naive $\mathrm{CD}^{+} \mathrm{T}$ cells is crucial in elucidating the mechanisms of Th17/ $\mathrm{Th}_{\mathrm{IL}-17}$ cell lineage differentiation.

Recently, Veldhoen et al. reported that Treg-derived TGF- $\beta$ induces the differentiation of $\mathrm{Th} 17 / \mathrm{Th}_{\mathrm{IL}-17}$ cells from naive $\mathrm{CD}^{+} \mathrm{T}$ cells in the presence of IL- 6 in vitro (Figure 1) (26). TGF- $\beta$-mediated Th17/Th ${ }_{\mathrm{IL}-17}$ cell differentiation is promoted by dendritic cell-derived IL- $1 \beta$ and TNF- $\alpha$. They showed that IL-23 is not essential for the development of Th17/Th ${ }_{\text {IL-17 }}$ cells, but required for their survival and expansion through the positive feedback loop that upregulates IL-6, IL- $1 \beta$, and TNF- $\alpha$. Thus, current evidence provides us with $2 \mathrm{Th} 17 / \mathrm{Th}_{\mathrm{IL}-17}$ cell differentiation pathways; one is IL-23 dependent and the other is IL-23 independent. Further studies are definitely required to address the precise roles of IL-23 and other factors in the development of Th17/Th $\mathrm{IL}-17$ cells in vivo.

\section{Concluding remarks}

While the importance of IL-12 in host defense against bacteria is widely accepted, the role of IL-23 in host defense is not well understood (Figure 2). Recent studies have revealed that IL12 and IL-23 have different roles in host defense. Mice deficient in IFN- $\gamma$, IFN- $\gamma$ R, or STAT 1 are highly susceptible to many different pathogens, including Leishmania major, Listeria monocytogenes, and Mycobacterium tuberculosis (27). IL-23 and IL-17 are also important in host defenses against infection. It should be noted that IL-12/IFN- $\gamma$ are primarily involved in host defenses against intracellular pathogens while IL-23/IL-17 are important for defenses against extracellular pathogens, including Klebsiella pneumoniae (28). This is because IFN- $\gamma$ stimulates the immune system to kill intracellular bacteria and infected host cells while IL-17 recruits and activates neutrophils. The detailed host defense mechanisms involving IL-23 and IL-17, however, still remain to be elucidated.

IL-17 is produced not only by $\mathrm{Th} 17 / \mathrm{Th}_{\mathrm{IL}-17}$ cells, but also by activated $\mathrm{CD}^{+} \mathrm{T}$ cells, $\mathrm{TCR} \gamma \delta^{+} \mathrm{T}$ cells, and neutrophils $(5,29)$. We have observed that CD4-CD8-TCR $\gamma \delta^{+} \mathrm{T}$ cells also produce IL-17 in IL-1 $\mathrm{Ra}^{-/}$mice, which spontaneously develop autoimmune arthritis (Komiyama et al., unpublished observations). Development of arthritis in these mice can be completely suppressed by IL-17 deficiency (6). Thus, IL-17 production by cells distinct from Th17/Th $\mathrm{TL}-17$ cells may also be involved in inflammatory responses and host defense mechanisms. It remains unclear, however, which of these producer cells are involved in the different allergic and infectious diseases and how the differentiation pathways of these cell lineages are controlled.

Taken together, accumulating evidence suggests that 3 independent pathways are involved in inflammatory responses: IL-12/IFN- $\gamma$, IL-4/IL-5/IL-13, and IL-23/ IL-17. These pathways are largely exclusive, although the effect of Th17/Th $\mathrm{IL}_{\mathrm{IL}}$ cells on Th1 and Th2 cells is not well understood. Identification of the major immune pathways responsible for the development of each disease is important for its treatment because suppression of 1 pathway may accelerate the others. Therapeutic targeting of the newly discovered IL-23/IL-17 immune axis may prove effective for the treatment of autoimmune and allergic inflammatory responses.

\section{Acknowledgments}

We would like to thank Shigeru Kakuta and Susumu Nakae (Stanford University School of Medicine, Stanford, California, USA) for critical reading of the manuscript.

Address correspondence to: Yoichiro Iwakura, Center for Experimental Medicine, Institute of Medical Science, University of Tokyo, 4-6-1 Shirokanedai, Minato-ku, Tokyo 108-8639, Japan. Phone: 81-3-5449-5536; Fax: 81-3-5449-5430; E-mail: iwakura@ims.u-tokyo.ac.jp.

1. Infante-Duarte, C., Horton, H.F., Byrne, M.C., and Kamradt, T. 2000. Microbial lipopeptides induce the production of IL-17 in Th cells. J. Immunol. 165:6107-6115

2. Harrington, L.E., et al. 2005. Interleukin 17-producing CD4+ effector $\mathrm{T}$ cells develop via a lineage distinct from the $\mathrm{T}$ helper type 1 and 2 lineages. Nat. Immunol. 6:1123-1132.

3. Langrish, C.L., et al. 2005. IL-23 drives a pathogenic $T$ cell population that induces autoimmune inflammation. J. Exp. Med. 201:233-240.

4. Park, H., et al. 2005. A distinct lineage of CD4 T cells regulates tissue inflammation by producing interleukin 17. Nat. Immunol. 6:1133-1141.

5. Kolls, J.K., and Linden, A. 2004. Interleukin-17 family members and inflammation. Immunity. 21:467-476.

6. Nakae, S., et al. 2003. IL-17 production from activated $\mathrm{T}$ cells is required for the spontaneous development of destructive arthritis in mice deficient in IL-1 receptor antagonist. Proc. Natl. Acad. Sci. U. S. A. 100:5986-5990.

7. Nakae, S., et al. 2002. Antigen-specific T cell sensitization is impaired in IL-17-deficient mice, causing suppression of allergic cellular and humoral responses. Immunity. 17:375-387.

8. Nakae, S., Nambu, A., Sudo, K., and Iwakura, Y. 2003. Suppression of immune induction of collagen-induced arthritis in IL-17-deficient mice. J. Immunol. 171:6173-6177.

9. Gately, M.K., et al. 1998. The interleukin-12/interleukin-12-receptor system: role in normal and pathologic immune responses. Annu. Rev. Immunol. 16:495-521.

10. Mannon, P.J., et al. 2004. Anti-interleukin-12 antibody for active Crohn's disease. N. Engl. J. Med. 351:2069-2079.

11. Bettelli, E., et al. 2004. Loss of T-bet, but not STAT1, prevents the development of experimental autoimmune encephalomyelitis. J. Exp. Med. 200:79-87. 
12. Hunter, C.A. 2005. New IL-12-family members: IL-23 and IL-27, cytokines with divergent functions. Nat. Rev. Immunol. 5:521-531.

13. McKenzie, B.S., Kastelein, R.A., and Cua, D.J. 2006. Understanding the IL-23-IL-17 immune pathway. Trends Immunol. 27:17-23.

14. Aggarwal, S., Ghilardi, N., Xie, M.H., de Sauvage, F.J., and Gurney, A.L. 2003. Interleukin-23 promotes a distinct CD4 $\mathrm{T}$ cell activation state characterized by the production of interleukin-17. J. Biol. Chem. 278:1910-1914.

15. Yen, D., et al. 2006. IL-23 is essential for T cellmediated colitis and promotes inflammation via IL-17 and IL-6. J. Clin. Invest. 116:1310-1316. doi:10.1172/JCI21404.

16. Strober, W., Fuss, I.J., and Blumberg, R.S. 2002. The immunology of mucosal models of inflammation. Annu. Rev. Immunol. 20:495-549.

17. Chen, Y., et al. 2006. Anti-IL-23 therapy inhibits multiple inflammatory pathways and ameliorates autoimmune encephalomyelitis. J. Clin. Invest. 116:1317-1326. doi:10.1172/JCI25308.

18. Cua, D.J., et al. 2003. Interleukin-23 rather than interleukin-12 is the critical cytokine for autoimmune inflammation of the brain. Nature 421:744-748.

19. Murphy, C.A., et al. 2003. Divergent pro- and antiinflammatory roles for IL-23 and IL-12 in joint autoimmune inflammation. J. Exp. Med. 198:1951-1957.

20. Afkarian, M., et al. 2002. T-bet is a STAT1-induced regulator of IL-12R expression in naive CD4+ T cells. Nat. Immunol. 3:549-557.

21. Wang, J., et al. 2006. Transcription factor T-bet regulates inflammatory arthritis through its function in dendritic cells. J. Clin. Invest. 116:414-421. doi:10.1172/JCI26631.

22. Parham, C., et al. 2002. A receptor for the heterodimeric cytokine IL-23 is composed of IL-12Rbeta 1 and a novel cytokine receptor subunit, IL-23R. J. Immunol. 168:5699-5708.

23. Kollias, G., and Kontoyiannis, D. 2002. Role of TNF/TNFR in autoimmunity: specific TNF receptor blockade may be advantageous to anti-TNF treatments. Cytokine Growth Factor Rev. 13:315-321.

24. Matsuki, T., Nakae, S., Sudo, K., Horai, R., and Iwakura, Y. 2006. Abnormal T cell activation caused by the imbalance of the IL-1/IL-1R antagonist system is responsible for the development of experimental autoimmune encephalomyelitis. Int. Immunol. 18:399-407.

25. Samoilova, E.B., Horton, J.L., Hilliard, B., Liu, T.S., and Chen, Y. 1998. IL-6-deficient mice are resistant to experimental autoimmune encephalomyelitis: roles of IL- 6 in the activation and differentiation of autoreactive T cells. J. Immunol. 161:6480-6486.

26. Veldhoen, M., Hocking, R.J., Atkins, C.J., Locksley, R.M., and Stockinger, B. 2006. TGFbeta in the context of an inflammatory cytokine milieu supports de novo differentiation of IL-17-producing T cells. Immunity. 24:179-189.

27. Shtrichman, R., and Samuel, C.E. 2001. The role of gamma interferon in antimicrobial immunity. Curr. Opin. Microbiol. 4:251-259.

28. Happel, K.I., et al. 2005. Divergent roles of IL-23 and IL-12 in host defense against Klebsiella pneumoniae. J. Exp. Med. 202:761-769.

29. Stark, M.A., et al. 2005. Phagocytosis of apoptotic neutrophils regulates granulopoiesis via IL-23 and IL-17. Immunity. 22:285-294.

\title{
Role of caveolin-1 in the regulation of the vascular shear stress response
}

\author{
Philippe G. Frank ${ }^{1,2,3}$ and Michael P. Lisanti²,3 \\ ${ }^{1}$ Department of Urology and ${ }^{2}$ Departments of Molecular Pharmacology and Medicine and the Albert Einstein \\ Diabetes Research and Training Center (DRTC), Albert Einstein College of Medicine, New York, New York, USA. \\ ${ }^{3}$ Department of Cancer Biology, Kimmel Cancer Center, Thomas Jefferson University, Philadelphia, Pennsylvania, USA.
}

\begin{abstract}
In blood vessels, endothelia are submitted to constant shear effects and are, under normal conditions, capable of responding to any variation in hemodynamic forces. Caveolae -50 - to 100 -nm plasma membrane invaginations present at the surface of terminally differentiated cells and particularly enriched in ECs - are composed of a high sphingolipid and cholesterol content and the protein caveolin-1 (Cav-1). Previous studies have suggested that caveolae and endothelial Cav-1 may regulate the vascular response to altered shear stress. In this issue of the JCI, Yu et al. have examined the role of Cav-1/caveolae in the regulation of flow-induced alterations (i.e., mechanotransduction) in vessels from wild-type mice, Cav-1-deficient mice, and Cav-1-deficient mice re-expressing Cav-1 only in ECs. Their data suggest that caveolae/Cav-1 may act as sensors of altered shear stress and that they also organize the signaling response in stimulated ECs (see the related article beginning on page 1284).
\end{abstract}

\section{Shear stress: an important regulator of endothelial cell function}

In the vasculature, blood vessels must respond rapidly to any external stimuli and especially to any physical change related to modifications in shear stress, which is a function of the blood viscosity and the velocity gradient at the arterial

Nonstandard abbreviations used: Cav-1, caveolin-1.

Conflict of interest: The authors have declared that no conflict of interest exists.

Citation for this article: J. Clin. Invest. 116:1222-1225 (2006). doi:10.1172/JCI28509. wall. In this context, blood vessels need to adapt and adjust their luminal diameters and their physical properties. ECs are the primary targets of these changes, as they are the first cell type exposed to these forces. One of the earliest findings that suggested an important role for ECs in this process was the observation of the ability of ECs to reorient and change shape during exposure to shear stress conditions. When submitted to steady laminar shear stress, ECs reorient in the direction of the flow and become remarkably elongated. These observations were made both in vitro (1) and in vivo $(2,3)$. These findings have suggested that ECs can respond and adapt to changes in blood flow. In fact, ECs act as sensors to transduce hydrodymanic forces. Not only does the morphology of ECs change, but other important signaling pathways have been shown to be regulated in response to altered shear stress. Several studies have shown that under laminar shear stress, the rate of EC proliferation is reduced compared with static conditions $(4,5)$. Oscillating and/or disturbed conditions have major effects on the pathology of the vasculature. In this regard, atherosclerotic lesions have been shown to develop primarily at sites of disturbed or altered blood flow, i.e., at bifurcations, branch ostia, and curved regions (6). Under oscillating and/or disturbed conditions, EC proliferation is increased compared with cells submitted to laminar shear stress and may allow ECs to repair injuries $(7,8)$. Other important regulatory pathways have now been shown to be activated under shear stress conditions (9-11). These pathways include those involved in changes in endothelial apoptotic, migratory, and permeability prop- 